\title{
The role of van der Waals forces in water adsorption on metals
}

\author{
Javier Carrasco ${ }^{1, a)}$, Jiří Klimeš ${ }^{2}$, and Angelos Michaelides ${ }^{3}$ \\ 1 Instituto de Catálisis y Petroleoquímica, \\ CSIC, Marie Curie 2, E-28049 Madrid, Spain \\ 2 Faculty of Physics and Center for Computational Nanoscience, \\ University of Vienna, Sensengasse 8/12, A-1090 Wien, Austria \\ 3 Thomas Young Centre, London Centre for Nanotechnology and Department of Chemistry, \\ University College London, London WC1E 6BT, UK \\ a) Electronic mail: j.carrasco@csic.es
}

\begin{abstract}
The interaction of water molecules with metal surfaces is typically weak and as a result van der Waals (vdW) forces can be expected to be of importance. Here we account for the systematic poor treatment of vdW forces in most popular density functional theory (DFT) exchange-correlation functionals by applying accurate non-local vdW density functionals. We have computed the adsorption of a variety of exemplar systems including water monomer adsorption on $\mathrm{Al}(111), \mathrm{Cu}(111)$, $\mathrm{Cu}(110), \operatorname{Ru}(0001), \operatorname{Rh}(111), \operatorname{Pd}(111), \operatorname{Ag}(111), \operatorname{Pt}(111)$, and unreconstructed $\mathrm{Au}(111)$, and small clusters (up to 6 waters) on $\mathrm{Cu}(110)$. We show that non-local correlations contribute substantially to the water-metal bond in all systems, whilst water-water bonding is much less affected by non-local correlations. Interestingly non-local correlations contribute more to the adsorption of water on the reactive transition metal substrates than they do on the noble metals. The relative stability, adsorption sites, and adsorption geometries of competing water adstructures rarely differ when comparing results obtained with semi-local functionals and the non-local vdW density functionals, which explains the previous success of semi-local functionals in characterizing adsorbed water structures on a number of metal surfaces.
\end{abstract}




\section{INTRODUCTION}

An accurate atomistic description of the water-solid interface is crucial for understanding many natural and technological processes such as atmospheric ice formation or fuel cell reactions. It is not surprising, therefore, that there has been an enormous amount of research devoted to this task in the past few decades ${ }^{1}-\underline{\underline{3}}$. In recent years, scanning probe techniques, in particular scanning tunneling microscopy (STM), have contributed significantly to the field by providing detailed insight into the structure and dynamics of water adstructures on the nanoscale. Generally such studies are, however, limited to well-defined, single crystal metal surfaces at low temperature and under ultra high vacuum (UHV) conditions $\underline{3} \underline{4}$. As a result the water-metal interface has become the workhorse system for understanding the basic chemistry and physics of how water interacts with solid substrates in general.

Computer simulation techniques, in particular density functional theory (DFT), have also played a central role in understanding the structure of water on metal surfaces 3.4 . DFT has, for example, been essential in unravelling the structure of water overlayers on $\operatorname{Pd}(111)^{\frac{5}{}}$, $\mathrm{Cu}(110)^{\underline{6}}$, and $\operatorname{Pt}(111)^{7}$. Notwithstanding the clear value of DFT in this area, the accuracy of the generalized gradient approximation (GGA) functionals, which are generally used in such studies, remains an important open question. This is mainly because the GGAs used fail to describe non-local van der Waals (vdW) dispersion forces, forces which are expected to be relevant in water adsorption and weak adsorption systems in general ${ }^{3,4,8,9}$. This casts a shadow over all standard density functional studies of water on metals and hampers progress in the field as it puts accurate predictions about interesting phenomena such as rates of heterogeneous ice nucleation beyond the reach of ab initio methods at present.

Recent years have seen a number of exciting developments with DFT based schemes for dealing with vdW dispersion forces (see e.g. $\operatorname{refs}^{10} \underline{-22}$ and for a recent review $\underline{23}$ ). With this has come a flurry of interest in understanding the role of vdW forces in water adsorption systems. In particular, recent dispersion-corrected DFT studies have been reported for water adsorption on $\mathrm{Rh}(111)^{25}, \mathrm{Cu}(110)^{26}, \operatorname{Pd}(111)^{27}, 28, \operatorname{Ag}(111)^{28}, \mathrm{Au}(111)^{28,29}$, and $\operatorname{Pt}(111)^{28}$ and by us on $\mathrm{Cu}(110)$ and $\mathrm{Ru}(0001)^{34}$. These studies have indeed indicated that vdW dispersion forces should be accounted for to properly describe the interaction of water molecules with the metal substrate underneath. In particular, it was shown that vdW dispersion forces explain a long standing discrepancy with respect to the relative thermodynamic stability of 
bulk ice and wetting layers on $\mathrm{Cu}$ and $\mathrm{Ru}$ surfaces ${ }^{34}$. However, despite this work, important issues remain poorly understood, such as the relative importance of vdW forces on different metal surfaces. Another open question is the role of vdW in determining the most stable adsorption site and adsorption structures for hydrogen bonded water clusters on metals.

Here we tackle these and related questions by exploring in detail the role of vdW dispersion forces in the adsorption of water monomers and clusters on a number of metal surfaces. The particular approach we use to treat vdW is the non-local van der Waals density functional (vdW-DF) of Langreth and Lundqvist and co-workers ${ }^{14}$ and some of its offspring ${ }^{19}$. These functionals from the vdW-DF family have shown great potential when applied to a number of systems where dispersion forces are important $19,22,24,30-42$. From the current study we find that non-local correlations contribute substantially to the adsorption of water monomers and clusters on metal surfaces in general. The contribution to the binding from non-local correlations varies from substrate to substrate and is actually greater on the more reactive transition metal surfaces than on the noble metals. Although vdW enhances the bonding with the substrate, the water-water interaction energies within the clusters remains largely unaffected by the inclusion of vdW dispersion forces. In addition the geometries of the water monomers and clusters on the various surfaces are not affected to any great extent by the inclusion of $\mathrm{vdW}$ dispersion forces, which explains the previous success ${ }^{3} \underline{\underline{4}}$ of GGA functionals in predicting adsorption structures of water on metals.

\section{METHODOLOGY AND COMPUTATIONAL DETAILS}

Density functional theory and supercell periodic models were used within the VASP 5.2 code $\underline{43}, \underline{4}$. Total energies and electron densities were computed with various different exchange-correlation functionals: (i) the semi-local Perdew-Burke-Ernzerhof (PBE) ${ }^{45}$; (ii) the non-local vdW-DF of Dion et al. $\stackrel{14}{*}$; and (iii) a couple of modified versions of the original vdW-DF, where the original GGA exchange functional has been replaced by an optimized PBE (optPBE) or optimized Becke88 (optB88) in order to improve the accuracy of the vdWDF scheme ${ }^{19}$. All vdW-DF calculations were carried out self-consistently within VASP as implemented by Klimeš et al $\underline{22}^{22}$ using the approach of Román-Pérez and Soler $\underline{46}$. In vdW-DF the exchange-correlation energy is calculated by adding three different terms: a GGA exchange energy, the local correlation energy obtained within the local density approximation 
(LDA), and a non-local correlation energy based on electron densities interacting via a model response function. In the original vdW-DF the GGA exchange is obtained with the revPBE functiona $\mathbb{1 4}^{47}$. However in ref. $\left[{ }^{[9}\right]$ alternative exchange functionals to revPBE were developed which can improve significantly the accuracy of the vdW-DF method. In all calculations the core electrons were replaced by projector augmented wave (PAW) potentials $\underline{49}$, whilst the wavefunctions of the valence electrons were expanded in plane-waves with a cut-off energy of $600 \mathrm{eV}$. PBE-based PAW potentials were used for all calculations. A Monkhorst-Pack ${ }^{50}$ grid with $12 \times 12 \times 1$ k-point sampling per $(1 \times 1)$ unit cell was used.

In the case of water monomer adsorption, the close-packed (111) and (0001) surfaces were modeled by $p(2 \times 2)$ unit cells, containing 6 atomic layers separated by at least $14 \AA$ of vacuum ( $23 \AA$ when computing binding curves). In the case of adsorbed water dimers on $\mathrm{Cu}(110)$ we considered a $p(3 \times 5)$ unit cell containing 4 atomic layers, whilst in the cases of adsorbed trimers, tetramers, pentamers, and hexamers a $p(4 \times 6)$ unit cell was employed. The metal atoms in the 3 ( 2 in the case of adsorbed water cluster models) bottom layers were fixed at their bulk-truncated positions during structure optimization procedures. We note that PBE lattice constants were used for all functionals. This is likely to influence very little the adsorption energies obtained with $\mathrm{vdW}$ functionals, since, for example, lattice constants are typically within 0.010 of $\mathrm{PBE}$ for optB88-vdW를. In all cases a dipole correction along the direction perpendicular to the metal surface was applied $\stackrel{51,52}{5}$ and geometry optimizations were performed with a residual force threshold of $0.015 \mathrm{eV} / \AA$.

Adsorption energies per water molecule were computed as follows:

$$
E_{\text {ads }}=\frac{E\left[\mathrm{H}_{2} \mathrm{O} / \mathrm{M}\right]-E[\mathrm{M}]-n E\left[\mathrm{H}_{2} \mathrm{O}\right]}{n},
$$

where $E\left[\mathrm{H}_{2} \mathrm{O} / \mathrm{M}\right]$ is the total energy of the adsorbed $n \mathrm{H}_{2} \mathrm{O}$ molecules, $E[\mathrm{M}]$ is the total energy of the relaxed bare metal slab and $E\left[\mathrm{H}_{2} \mathrm{O}\right]$ is the total energy of an isolated gas phase $\mathrm{H}_{2} \mathrm{O}$ molecule. Within this definition a negative adsorption energy corresponds to a favorable (exothermic) adsorption process.

In order to analyze the role of van der Waals dispersion forces on adsorption, we have decomposed $E_{\text {ads }}$ in to different energy contributions ${ }^{53}$. We define the water-water contribution, $E_{\text {gas }}^{\mathrm{ww}}$, to $E_{\text {ads }}$ as: 


$$
E_{\mathrm{gas}}^{\mathrm{ww}}=\frac{E^{\mathrm{tot}}\left[n \mathrm{H}_{2} \mathrm{O}\right]-n E^{\mathrm{tot}}\left[\mathrm{H}_{2} \mathrm{O}\right]}{n}
$$

where $E^{\text {tot }}\left[n \mathrm{H}_{2} \mathrm{O}\right]$ is the total energy of the water structure in the absence of the substrate, but with all atoms fixed in the precise geometries they adopt in the adsorption structure. Notwithstanding the fact that any energy decomposition scheme is to some extent arbitrary ${ }^{53}$, the estimate of the water-metal bonding, $E_{\mathrm{ads}}^{\mathrm{wm}}$, is simply taken as the difference between $E_{\text {ads }}$ and $E_{\text {gas }}^{\text {ww }}$. We have also examined the non-local correlation part, $E^{\text {nlc }}$, of the exchange-correlation energy which is obtained directly from the calculation. We note that $E^{\text {nlc }}$ includes a part which acts as a semi-local correction to LDA correlation, i.e. in a similar spirit as PBE correlation works. Thus the "non-local" interaction means interaction beyond LDA correlation. The corresponding attraction due to the non-local correlation in the adsorption energy per water molecule can be calculated as:

$$
E_{\mathrm{ads}}^{\mathrm{nlc}}=\frac{E^{\mathrm{nlc}}\left[\mathrm{H}_{2} \mathrm{O} / \mathrm{M}\right]-E^{\mathrm{nlc}}[\mathrm{M}]-n E^{\mathrm{nlc}}\left[\mathrm{H}_{2} \mathrm{O}\right]}{n}
$$

an analogous expression to Eq. 1, but where total energies are substituted by their non-local correlation part.

\section{RESULTS AND DISCUSSION}

\section{A. Adsorbed water monomers}

First we consider the adsorption of an isolated water monomer on a series of metal surfaces. This is the simplest water adsorption system possible to investigate the role of vdW interactions with the metal, since no H-bonds between water molecules are present. In the first place we explored the effect of vdW interactions in determining the most stable adsorption site on a selection of close-packed metal surfaces. Considering the most stable adsorption site, we then extended the study to a larger number of substrates to investigate in a systematic manner the dependence of $\mathrm{vdW}$ interactions with the nature of the metal.

Previous DFT studies with semi-local functionals have predicted that water adsorbs on atop sites on close-packed surfaces $\underline{54}-\underline{57}$. Since recent studies have shown that vdW forces can alter adsorption structures $\underline{39}, \underline{50}$, it is important to establish if vdW changes the preferred site for water adsorption on metals. To this end we computed the optB88-vdW (and for 
reference PBE) adsorption energies of a water monomer on $\mathrm{Ag}(111), \mathrm{Au}(111)$, and $\mathrm{Ru}(0001)$. More than 30 different adsorption sites and molecule orientations were explored, from which 6 stable and representative structures are shown in Fig. 1. We found that the most stable adsorption site with the optB88-vdW functional is the atop site, consistent with previous work and our own new PBE calculations reported in Table I. These results indicate that accounting for vdW interactions does not lead to a qualitative change in the mechanism governing the water-metal adsorption geometry of adsorbed monomers $\frac{57}{}$. Nevertheless, vdW interactions increase the strength of the bond with the substrate. In particular, on the noble metals, orientations of the water molecule with the oxygen atom away from the surface (S5 and S6) have almost no binding to the surface at the PBE level, whereas optB88-vdW adsorption energies are relatively large (Table I). This will very likely be of relevance to water monomer diffusion, with the diffusing water molecule able to access many more stable configurations when dispersion forces are accounted for.

In order to gain more insight into the role of vdW forces on the adsorption energy and their dependence on the specific functional chosen, we calculated the PBE, revPBEvdW, optPBE-vdW, and optB88-vdW binding curves of a water monomer on $\mathrm{Ag}(111)$ and $\mathrm{Ru}(0001)$ as a function of the distance between oxygen and the metal atom underneath (Fig. 2). Each point in the graph is obtained by keeping the $z$ coordinate of the water oxygen atom fixed while relaxing the rest of the atoms in the system except the 3 bottom layers of the metal slab. Of the functionals considered, PBE predicts the weakest interaction at longrange. Although revPBE-vdW recovers the long-range attraction, the interaction strength around the equilibrium distance is only slightly larger than that obtained from PBE on $\mathrm{Ag}$ and on $\mathrm{Ru}$ the revPBE-vdW binding minimum is actually shallower than that obtained with PBE. At long-range, the energies of the optPBE-vdW and optB88-vdW functionals are similar to revPBE-vdW, but on approaching the surface become significantly more attractive. Computing the non-local correlation contribution to the total adsorption energy reveals that this is indeed the leading attractive term between the water molecule and the surface as observed previously by Hamada and co-workers 25.

Considering the most stable adsorption site and adsorbed water orientation identified previously (S1), we examine now the adsorption energy of water monomers on a large range of metal substrates: $\mathrm{Al}(111), \mathrm{Cu}(111), \mathrm{Cu}(110), \mathrm{Ru}(0001), \mathrm{Rh}(111), \operatorname{Pd}(111), \operatorname{Ag}(111)$, $\mathrm{Pt}(111)$, and unreconstructed $\mathrm{Au}(111)$. Also included in this systematic study are results 
from the original revPBE-vdW functional for comparative purposes with respect to optB88vdW. The computed adsorption energies and optimized distances are summarized in Table II] A key observation is that revPBE-vdW does not always enhance adsorption energies with respect to PBE. Indeed the revPBE-vdW adsorption energies can either be larger or smaller than PBE. This is consistent with previous studies for various adsorption systems where the revPBE-vdW adsorption energies can either be similar to or slightly smaller than PBE $14,19,59,61-65$. This behaviour is a direct consequence of the underlying overly repulsive revPBE exchange in the revPBE-vdW kernel, which also causes pure revPBE adsorption energies to be underestimated compared to PBE. Indeed water-metal distances $\left(d_{\mathrm{w}-\mathrm{m}}\right)-$ defined here as the distance between the $\mathrm{O}$ atom of a water molecule and the nearest metal atom on the surface - optimized with revPBE-vdW are substantially larger (up to $0.32 \AA$ ) than those obtained from PBE. Another key observation is that optB88-vdW consistently provides larger adsorption energies than PBE and similar water-metal distances (within $0.1 \AA$ ) for all investigated systems. As observed before in the binding curves of $\operatorname{Ag}(111)$ and $\mathrm{Ru}(0001)$, we see that non-local correlation $\left(E_{a d s}^{\text {nlc }}\right)$ is again the principal attractive contribution to the total adsorption energy $\left(E_{a d s}\right)$ as shown in Table II. Specifically, $E_{a d s}^{\text {nlc }}$ is in most cases considerably larger than $E_{a d s}$ at the equilibrium geometry independent of the metal and functionals considered. Although the contribution of vdW dispersion forces to the non-local correlation at equilibrium distances is probably not dominant, it clearly enhances the total adsorption energy as indicated by the fact that typical optB88-vdW adsorption energies are in general 110-190 meV/ $\mathrm{H}_{2} \mathrm{O}$ larger than PBE values (Table II). Interestingly, larger adsorption energy enhancements are found on more reactive metals than on noble ones. For example, when comparing $\operatorname{Pd}(111)$ and $\operatorname{Ag}(111)$, the difference between PBE and optB88-vdW adsorption energies is $180 \mathrm{meV} / \mathrm{H}_{2} \mathrm{O}$ and $144 \mathrm{meV} / \mathrm{H}_{2} \mathrm{O}$, respectively. Similarly, the vdW enhancement is also larger when comparing the adsorption on $\mathrm{Pt}(111)$ and $\mathrm{Au}(111): 186 \mathrm{meV} / \mathrm{H}_{2} \mathrm{O}$ and $158 \mathrm{meV} / \mathrm{H}_{2} \mathrm{O}$, respectively. This behavior can be rationalized in terms of the equilibrium water-metal distance in each case as discussed recently by Liu et al. for benzene adsorption on metal surfaces $\frac{65}{}$. As shown in Table $\amalg$ the water molecule adsorbs closer to the surface on reactive metals such as $\mathrm{Pd}$ and $\mathrm{Pt}$ than it does on more noble substrates such as Ag and Au. The shorter water-metal distances lead to larger vdW dispersion forces and, therefore, larger total adsorption energies.

Overall we have shown that optB88-vdW (and for the systems considered optPBE-vdW) 
increases adsorption energies by accounting for vdW interactions without substantially altering adsorption sites or bonding distances with respect to PBE. In contrast, although revPBE-vdW is able to catch similarly vdW interactions at long distances, adsorption energies are not increased beyond those obtained with PBE. Unfortunately, experimental data is not yet available against which we can benchmark our computed adsorption energies. This is mainly because unlike simpler adsorption systems such as CO or noble gas adsorption, water molecules readily diffuse and cluster on the surface, forming complex cluster and overlayer structures (e.g. the clusters discussed in section IIIB). Clusters and overlayers obviously complicate the analysis of experimental adsorption enthalpy determinations, especially with approaches such as temperature programmed desorption ${ }^{66}$. Although experimental adsorption energies for water monomers on well-defined metal surfaces are lacking, we note that in a recent study it was possible to perform the first single crystal adsorption microcalorimetry measurements of the energy of a well-dened water adstructure (a partially dissociated water adlayer stabilized by hydrogen bonding $)^{37}$. For this one overlayer structure we were able to show that the inclusion of vdW forces using optB88-vdW was crucial for achieving quantitative agreement with experiment ${ }^{37}$; a similar conclusion has also been drawn for the adsorption of benzene on $\mathrm{Au}(111)$ and $\mathrm{Pt}(111)^{65}$. However, in general, accurate experimental measurements of adsorption energies of water on well-defined surfaces are severely lacking and urgently needed $\stackrel{4}{4} 23$.

\section{B. Adsorbed water clusters}

Let us focus now on more complex water adsorption systems than simple adsorbed water monomers, in which we simultaneously consider the interplay of water-metal and waterwater interactions. To this end we have considered a series of adsorbed water clusters with between 2 and 6 water molecules on $\mathrm{Cu}(110)$. We have considered $\mathrm{Cu}(110)$ because of a number of recent STM experiments for low coverages of water on this surface ${ }^{6,26,67-69}$ and because the open (110) surface allows us to explore a rich variety of isomers for a given water cluster and, in so doing, analyze the role of vdW in determining the relative energies of various adsorbed water clusters.

For each of the clusters examined we considered a range of different initial geometries which upon optimization lead to a number of stable or metastable water clusters, as shown in 
Fig. 3. In Table [1] we summarize the adsorption energies of all these structures employing various functionals. For the adsorbed trimers and tetramers our results are in general agreement with a recent study by Kumagai et al $\underline{\underline{26}}$, who by combining STM and DFT identified chain trimers and cyclic tetramers as the most stable species.

Consistent with the results of the water monomers we find for the clusters that including vdW interactions through optB88-vdW leads to a substantial increase $\left(>120 \mathrm{meV} / \mathrm{H}_{2} \mathrm{O}\right)$ of the total adsorption energy when compared with PBE. The optPBE-vdW adsorption energies are typically smaller than those from optB88-vdW by $\approx 50 \mathrm{meV} / \mathrm{H}_{2} \mathrm{O}$ and revPBEvdW values are in all cases less than PBE by at least $45 \mathrm{meV} / \mathrm{H}_{2} \mathrm{O}$. Although in most situations the structures obtained with PBE and optB88-vdW are similar, there are some occasions when accounting for van der Waals dispersion forces can alter the relative stabilities of the clusters considered. In particular, optB88-vdW tends to favor planar structures over buckled ones. For example, in the case of water tetramers the buckled cluster (Te-I) is preferred at the PBE or even revPBE-vdW level, over the planar tetramer (Te-II) by about $10-15 \mathrm{meV} / \mathrm{H}_{2} \mathrm{O}$. However, the situation is reversed when considering optB88-vdW which predicts the planar tetramer (Te-II) to be $15 \mathrm{meV} / \mathrm{H}_{2} \mathrm{O}$ more stable than the buckled one. It turns out that this result can explain the absence of corrugation in the STM images of Kumagai et al. $\underline{\underline{26}}$. The lack of buckling was attributed to tip-induced reorientation or fast dynamical fluctuations between the two isomers ${ }^{26}$. However, here we see that there is no need to invoke such arguments as the most stable structure when vdW is accounted for is planar. Similarly, the preference for planar adstructures when switching on vdW dispersion through optB88-vdW is again observed in the case of the P-II and P-III pentamers, and the H-II and H-III hexamers (Table III).

Another important observation is that the clusters become more stable as the clusters increase in size (Fig. 4). This is consistent with what has been observed on other surfaces 55,70 and gas phase clusters 1 , $\underline{72}$ and is due to the formation of $\mathrm{H}$-bonds between water molecules and to a well-known cooperative effect, i.e., the increase of the number of $\mathrm{H}$-bonds within the clusters results in stronger H-bonds. Interestingly, the nature of this cooperative effect shows a very small dependence with respect to the particular functional considered: both PBE and the vdW-DFs present similar trends, only the magnitude of the total adsorption energy is shifted (Fig. 4). We see, therefore, that vdW forces seem to play a minor role in describing cooperative water-water effects in these systems. 
In order to gain deeper insight into these adsorption systems, we decomposed the total adsorption energy into water-water and water-metal bonding contributions as given by Eq. 2. As shown in Table III, the water-water bonding contribution to the adsorption energy obtained from the various functionals is very similar: the PBE, optPBE-vdW, and optB88vdW results are all within $13 \mathrm{meV} / \mathrm{H}_{2} \mathrm{O}$ for all adsorption systems (the revPBE-vdW waterwater interactions are systematically smaller in all cases). It is clear, therefore, that the main effect of vdW forces on the total interaction comes from the water-metal bonding. Indeed the approximately $120 \mathrm{meV} / \mathrm{H}_{2} \mathrm{O}$ increase in the adsorption energy comes almost exclusively from the water-metal bonding. This is consistent with our earlier work on icelike films on $\mathrm{Ru}$ and $\mathrm{Cu}^{34}$. Unlike the GGA functionals where the contribution to the binding comes from density overlap, the non-local functionals give binding even between non-overlapping electron densities ${ }^{73}$. Therefore, when considering non-local functionals not only a small overlap region gives contribution to the adsorption energy, but a larger part of the surface too. This leads to a stronger water-metal interaction for non-local functionals than GGA functionals. Moreover, this also explains that the shift in the adsorption energy caused by the vdW density functional with respect to $\mathrm{PBE}$ is almost constant between different clusters. In general, water-metal bonding is greater than water-water bonding and is responsible for the relative stability of the isomers and preference for more planar rather than buckled isomers, independent of the considered functional. Upon comparing the strength of the water-water interaction for the various clusters considered, we find that the water-water bonding is strongest in the water tetramers (Te-I and Te-II). Larger clusters have larger total adsorption energies, but their water-water bonding is substantially reduced with respect to the optimal H-bond configuration offered by a tetragonal arrangement on the $\mathrm{Cu}(110)$ surface. This becomes especially evident in the case of pentamers, where the most stable isomer, P-I, shows actually a relatively small optB88-vdW water-water bonding $\left(-169 \mathrm{meV} / \mathrm{H}_{2} \mathrm{O}\right)$ when compared for example to isomer $\mathrm{P}-\mathrm{V}\left(-260 \mathrm{meV} / \mathrm{H}_{2} \mathrm{O}\right)$.

Regarding the effect of vdW forces on the adsorption geometry of the clusters, we summarize in Table [V] and Fig. 5 the averaged water-metal and water-water distances computed with different functionals. Essentially PBE geometries do not differ significantly with respect to the optB88-vdW or optPBE-vdW functionals for both water-metal and water-water distances. All distances are within $0.05 \AA$ of $\mathrm{PBE}$ for optB88-vdW and within $0.09 \AA$ of PBE for optPBE-vdW. On the contrary, revPBE-vdW predicts larger water-metal $(<0.15$ 
$\AA)$ and water-water $(<0.14 \AA)$ distances. It is interesting, therefore, that despite a noticeable enhancement in water-metal bonding when vdW is accounted for, the water-metal distances remain rather similar.

\section{CONCLUSION}

We have investigated the role of vdW dispersion forces in water-metal bonding by considering different non-local vdW-DFs and a range of water monomers and small water clusters on a series of metal surfaces. Analysis of our results reveals an enhancement of adsorption energies (typically $>110 \mathrm{meV} / \mathrm{H}_{2} \mathrm{O}$ ) due to $\mathrm{vdW}$ interactions with respect to the widely used PBE functional. The increase in the adsorption energy comes almost exclusively from an increased water-metal interaction, with the water-water interaction within the adsorbed clusters being essentially unaffected by the inclusion of vdW dispersion forces. As a consequence, we observe that in general the explicit consideration of vdW dispersion forces does not alter the relative stabilities of structures predicted by PBE. In addition, despite increases in the total adsorption energies, the adsorption sites remain unchanged and adsorption geometries (water-metal bond and water H-bond lengths) are very similar when comparing PBE and optB88-vdW in general. On the few occasions when the PBE and optB88-vdW structures differ, the optB88-vdW structures are flatter with any high-lying water molecules brought closer to the surface, which for the particular case of the water tetramer on $\mathrm{Cu}(110)$ produces an adsorption structure in better agreement with experiment. This is a direct consequence of the fact that non-local correlation is enhanced by shorter water-metal distances. It is interesting to note that the general similarity between the structures obtained with and without vdW dispersion forces is in contrast to what has been found for gas phase water clusters. In particular for the water hexamers - where this issue has been considered in greatest detail - the relative energies of the relevant low energy isomer structures differ completely depending on whether vdW is accounted for or not $\underline{72}$.

\section{ACKNOWLEDGEMENTS}

JC is a Ramón y Cajal fellow and Newton Alumnus supported by the Spanish Government and The Royal Society, respectively. AM is supported by the European Research Council and 
the Royal Society through a Royal Society Wolfson Research Merit Award. We are grateful for computer time to UCL Research Computing, the London Centre for Nanotechnology, and the UK's national high performance computing service HECToR (from which access was obtained via the UK's Material Chemistry Consortium, EP/F067496).

1 P. A. Thiel, T. E. Madey, Surf. Sci. Rep. 7, 211 (1987).

2 M. A. Henderson, Surf. Sci. Rep. 46, 1 (2002).

3 A. Hodgson and S. Haq, Surf. Sci. Rep. 64, 381 (2009).

4 J. Carrasco, A. Hodgson, A. Michaelides, Nature Mater. 11, 667 (2012).

5 J. Cerda, A. Michaelides, M. L. Bocquet, P. J. Feibelman, T. Mitsui, M. Rose, E. Formin, E. M. Salmeron, Phys. Rev. Lett. 93, 116101 (2004).

6 J. Carrasco, A. Michaelides, M. Forster, S. Haq, R. Raval, A. Hodgson, Nature Mater. 8, 427 (2009).

7 S. Nie, P. J. Feibelman, N. C. Bartelt, and K. Thürmer, Phys. Rev. Lett. 105, 026102 (2010).

8 P. J. Feibelman, Phys. Chem. Chem. Phys. 10, 4688 (2008).

9 P. J. Feibelman, Faraday Discuss. 141, 467 (2009).

10 B. Santra, A. Michaelides, M. Fuchs, A. Tkatchenko, C. Filippi, M. Scheffler, J. Chem. Phys. 129, 194111 (2008).

11 Q. Wu, W. Yang, J. Chem. Phys. 116, 515 (2002).

12 S. Grimme, J. Comput. Chem. 25, 1463 (2004).

13 P. Jurečka, J. Čverny̌, P. Hobza, D. R. Salahub, J. Comput. Chem. 28, 555 (2007).

14 M. Dion, H. Rydberg, E. Schröder, D. C. Langreth, B. I. Lundqvist, Phys. Rev. Lett. 92, 246401 (2004).

15 A. D. Becke, E. R. Johnson, J. Chem. Phys. 122, 154104 (2005).

16 P. L. Silvestrelli, Phys. Rev. Lett. 100, 053002 (2008).

17 O. A. von Lilienfeld, I. Tavernelli, U. Rothlisberger, D. Sebastiani, Phys. Rev. Lett. 93, 153004 (2004).

18 A. Tkatchenko and M. Scheffler, Phys. Rev. Lett. 102, 073005 (2009).

19 J. Klimeš, D. R. Bowler, and A. Michaelides, J. Phys.: Condens. Matter 22, 022201 (2010).

20 L. Schimka, J. Harl, A. Stroppa, A. Grüneis, M. Marsman, F. Mittendorfer, and G. Kresse, 
Nature Mater. 9, 741 (2010).

21 O. A. Vydrov, T. Van Voorhis, J. Chem. Phys. 133, 244103 (2010).

22 J. Klimeš, D. R. Bowler, and A. Michaelides, Phys. Rev. B 83, 195131 (2011).

23 J. Klimeš, A. Michaelides, J. Chem. Phys. 137, 120901 (2012).

24 D. C. Langreth, B. I. Lundqvist, S. D. Chakarova-Käck, V. R. Cooper, M. Dion, P. Hyldgaard, A. Kelkkanen, J. Kleis, Lingzhu Kong, Shen Li, P. G. Moses, E. Murray, A. Puzder, H. Rydberg, E. Schröder, and T. Thonhauser, J. Phys.: Condens. Matter. 21, 084203 (2009).

25 I. Hamada, K. Lee, Y. Morikawa, Phys. Rev. B 81, 115452 (2010).

26 T. Kumagai, H. Okuyama, S. Hatta, T. Aruga, and I. Hamada, J. Chem. Phys. 134, 024703 (2011).

27 A. Poissier, S. Ganeshan, M. V. Fernández-Serra, Phys. Chem. Chem. Phys. 13, 3375 (2011).

28 K. Tonigold and A. Gross, J. Comp. Chem. 33, 695 (2012).

29 R. Nadler and J. F. Sanz, J. Chem. Phys. 137, 114709 (2012).

30 A. K. Kelkkanen, B. I. Lundqvist, and J. K. Nørskov, Phys. Rev. B 83, 113401 (2011).

31 Y. N. Zhang, F. Hanke, V. Bartolani, M. Persson, R, Q. Wu, Phys. Rev. Lett. 106, 236103 (2011).

32 B. Santra, J. Klimeš, D. Alfè, A. Tkatchenko, B. Slater, A. Michaelides, R. Car, and M. Scheffler, Phys. Rev. Lett. 107, 185701 (2011).

33 X. L. Hu, J. Carrasco, J. Klimeš, and A. Michaelides, Phys. Chem. Chem. Phys. 13, 12447 (2011).

34 J. Carrasco, B. Santra, J. Klimeš, and A. Michaelides, Phys. Rev. Lett. 106, 026101 (2011).

35 M. A. F. Addato, A. A. Rubert, G. A. Benítez, M. H. Fonticelli, J. Carrasco, P. Carro, R. C. Salvarezza, J. Phys. Chem. C 115, 17788 (2011).

36 T. J. Lawton, J. Carrasco, A. E. Baber, A. Michaelides, and E. C. H. Sykes, Phys. Rev. Lett. 107, $256101(2011)$.

37 W. Lew, M. C. Crowe, C. T. Campbell, J. Carrasco, and A. Michaelides, J. Chem. Phys. C 115, 23008 (2011).

38 P. L. Silvestrelli, A. Ambrosetti, S. Grubisic, and F. Ancilotto, Phys. Rev. B 85, 165405 (2012).

39 G. Li, I. Tamblyn, V. R. Cooper, H.-J. Gao, and J. B. Neaton, Phys. Rev. B 85, 121409 (2012).

40 T. J. Lawton, J. Carrasco, A. E. Baber, A. Michaelides, and E. C. H. Sykes, Phys. Chem. Chem. Phys. 14, 11846 (2012). 
41 D. Fernández-Torre, K. Kośmider, J. Carrasco, M. V. Ganduglia-Pirovano, and R. Pérez, J. Phys. Chem. C 116, 13584 (2012).

42 M. Forster, R. Raval, J. Carrasco, A. Michaelides, and A. Hodgson, Chem. Sci. 3, 93 (2012).

43 G. Kresse, J. Hafner, Phys. Rev. B 47, 558 (1993).

44 G. Kresse, J. Furthmüller, Phys. Rev. B 54, 11169 (1996).

45 J. P. Perdew, K. Burke, M. Ernzerhof, Phys. Rev. Lett. 77, 3865 (1996); 78, 1396 (1997).

46 G. Román-Pérez and J. M. Soler, Phys. Rev. Lett. 103, 096102 (2009).

47 Y. Zhang and W. Yang, Phys. Rev. Lett. 103, 063004 (2009).

48 P. Jurečka, J. Šponer, J. Černý, and P. Hobza, Phys. Chem. Chem. Phys. 8, 1985 (2006).

49 G. Kresse, D. Joubert, Phys. Rev. B 59, 1758 (1999).

50 H. J. Monkhorst, J. D. Pack, Phys. Rev. B 13, 5188 (1976).

51 G. Makov and M. C. Payne, Phys. Rev. B 51, 4014 (1995).

52 J. Neugebauer and M. Scheffler, Phys. Rev. B 46, 16967 (1992).

53 A. Michaelides, A. Alavi, and D. A. King, Phys. Rev. B 69, 113404 (2004).

54 A. Michaelides, V. A. Ranea, P. L. de Andres, D. A. King, Phys. Rev. Lett. 90, 216102 (2003).

55 S. Meng, E. G. Wang, S. Gao, Phys. Rev. B 69, 195404 (2004).

56 J. Knudsen, A. U. Nilekar, R. T. Vang, J. Schnadt, E. L. Kunkes, J. A. Dumesic, M. Mavrikakis, F. Besenbacher, J. Am. Chem. Soc. 129, 6485 (2007).

57 J. Carrasco, A. Michaelides, and M. Scheffler, J. Chem. Phys. 130, 184707 (2009).

58 N. Atodiresei, V. Caciuc, P. Lazić, and S. Bügel, Phys. Rev. Lett. 102, 136809 (2009).

59 F. Mittendorfer, A. Garhofer, J. Redinger, J. Klimeš, J. Harl, and G. Kresse, Phys. Rev. B 84, 201410 (2011).

60 W. Liu, A. Savara, X. Ren, W. Ludwig, K.-H. Dostert, S. Schauermann, A. Tkatchenko, H.-J. Freund, and M. Scheffler, J. Phys. Chem. Lett. 3, 582 (2012).

61 A. Puzder, M. Dion, and D. C. Langreth, J. Chem. Phys. 124, 164105 (2006).

62 E. Ziambaras, J. Kleis, E. Schröder, and P. Hyldgaard, Phys. Rev. B 76, 155425 (2007).

63 K. Lee, E. D. Murray, L. Kong, B. I. Lundqvist, and D. C. Langreth, Phys. Rev. B 82, 081101 (2010).

64 I. Hamada, J. Chem. Phys. 133, 214503 (2010).

65 W. Liu, J. Carrasco, B. Santra, A. Michaelides, M. Scheffler, A. Tkatchenko, Phys. Rev. B 86, $245405(2012)$. 
66 G. S. Karlberg, G. Wahnstrom, C. Clay, G. Zimbitas, and A. Hodgson, J. Chem. Phys. 124, $204712(2006)$.

67 T. Yamada, S. Tamamori, and H. Okuyama, Phys. Rev. Lett. 96, 036105 (2006).

68 J. Lee, D. C. Sorescu, K. D. Jordan, and J. T. Jr. Yates, J. Phys. Chem. C 112, 17672 (2008).

69 M. Forster, R. Raval, A. Hodgson, J. Carrasco, and A. Michaelides, Phys. Rev. Lett. 106, 046103 (2011).

70 A. Michaelides, Faraday Discuss. 136, 287 (2007).

71 K. Liu, J. D. Cruzan, and R. J. Saykally, Science 271, 929 (1996).

72 B. Santra, A. Michaelides, and M. Scheffler, J. Chem. Phys. 127, 184104 (2007).

73 P. Lazić, N. Atodiresei, R. Brako, B. Gumhalter, and S. Blügel, J. Phys.: Condens. Matter 24, 424215 (2012). 

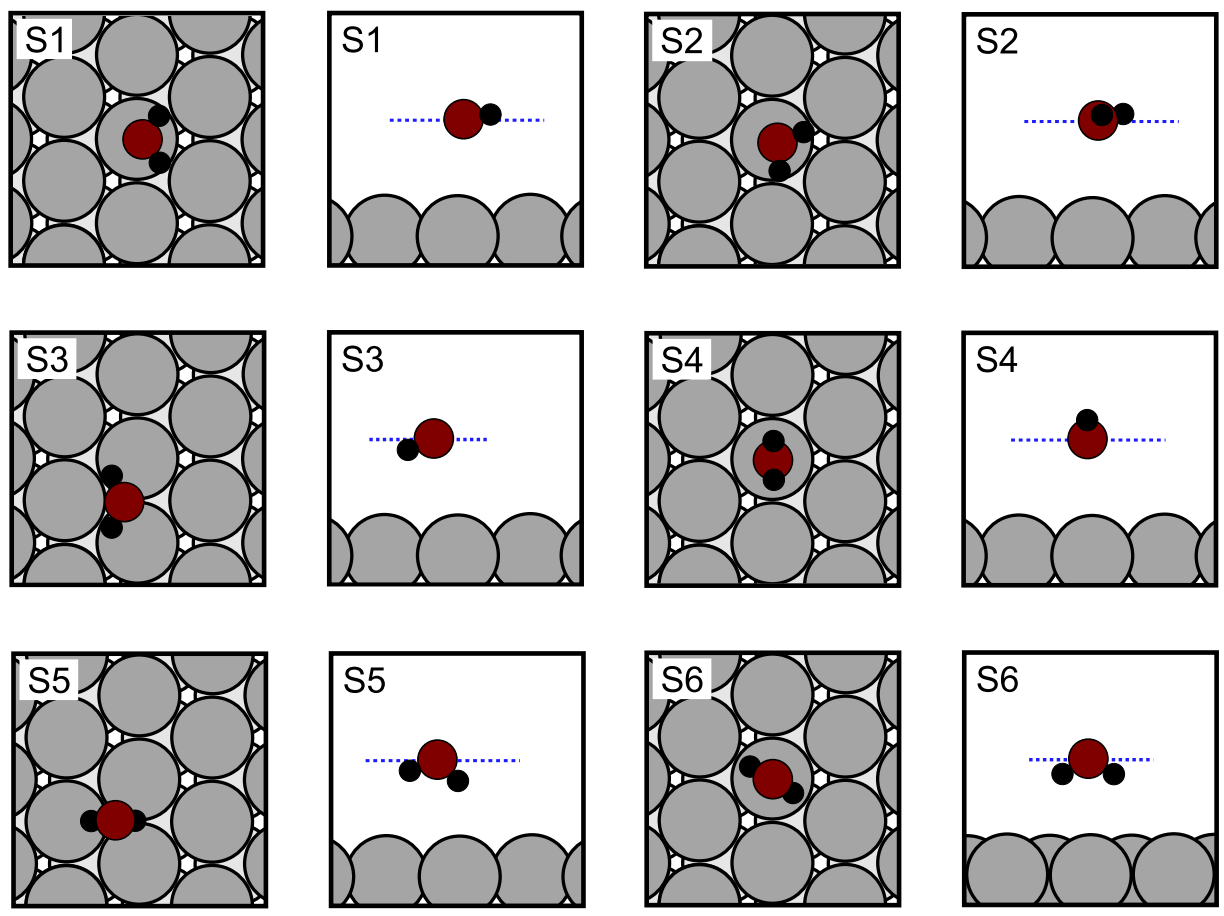

FIG. 1: Top and side views of water monomers adsorbed at different sites on a close-packed metal surface. Small black, red, and grey spheres stand for H, O, and metal atoms, respectively. 

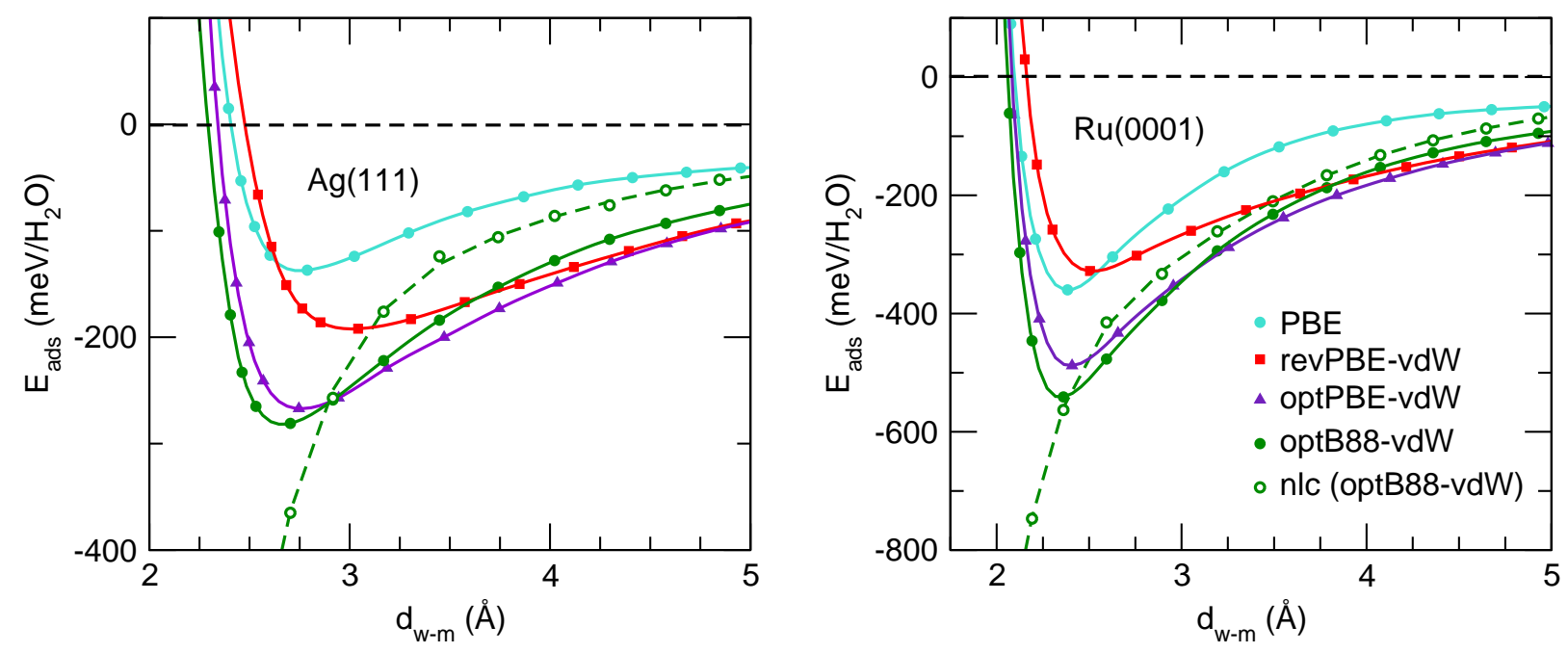

FIG. 2: Adsorption energy of $\mathrm{H}_{2} \mathrm{O}$ monomer on $\mathrm{Ag}(111)$ and $\mathrm{Ru}(0001)$ as a function of the vertical water-metal distance $\left(d_{\mathrm{w}-\mathrm{m}}\right)$, defined as the distance between the $\mathrm{O}$ atom of a water molecule and the nearest metal atom on the surface. Four different functionals are considered and the optB88vdW non-local correlation (nlc) contribution to the total energy (Eq. 3) is also shown (open circles and dashed line). The lines are merely a guide to the eye. 

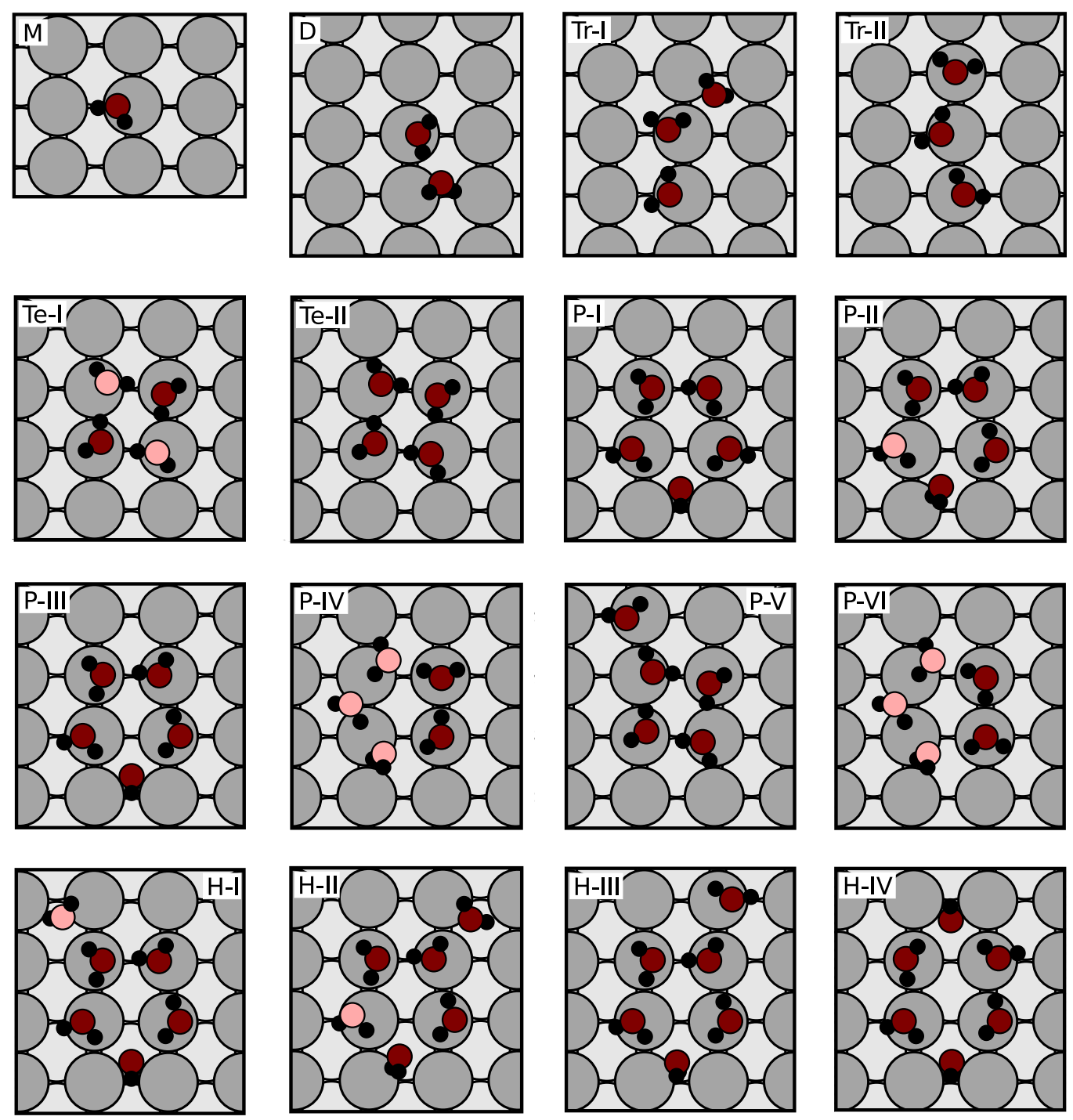

FIG. 3: Top view of water monomer (M), dimer (D), trimers (Tr-I and Tr-II), tetramers (Te-I and Te-II), pentamers (P-I, P-II, P-III, P-IV, P-V, and P-VI), and hexamers (H-I, H-II, H-III, and H-IV) adsorbed on $\mathrm{Cu}(110)$. Small black, red, dark grey, and light grey spheres stand for H, O, and $\mathrm{Cu}$ in the first and second layer, respectively. Light red spheres indicate water molecules that are relatively far away from the metal surface (typically $>3 \AA$ ). 


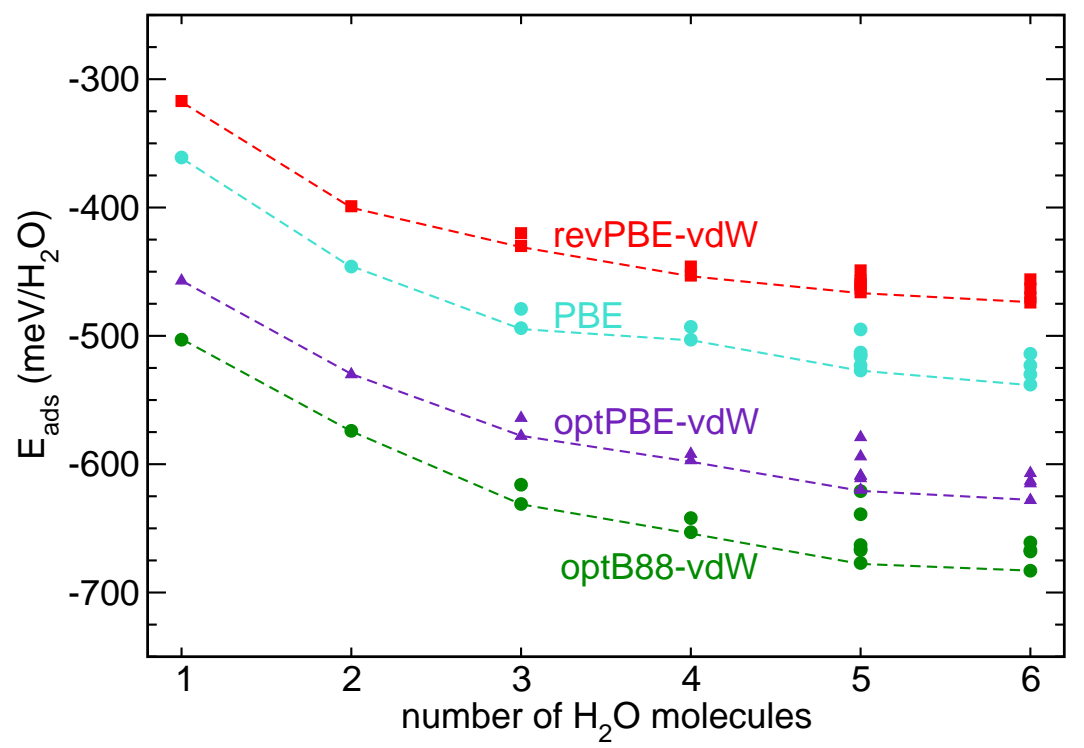

FIG. 4: Adsorption energies of different sized water clusters adsorbed on $\mathrm{Cu}(110)$ using $\mathrm{PBE}$, revPBE-vdW, optPBE-vdW, and optB88-vdW. Dashed lines connect the most stable isomers for a given number of $\mathrm{H}_{2} \mathrm{O}$ molecules with each functional. 

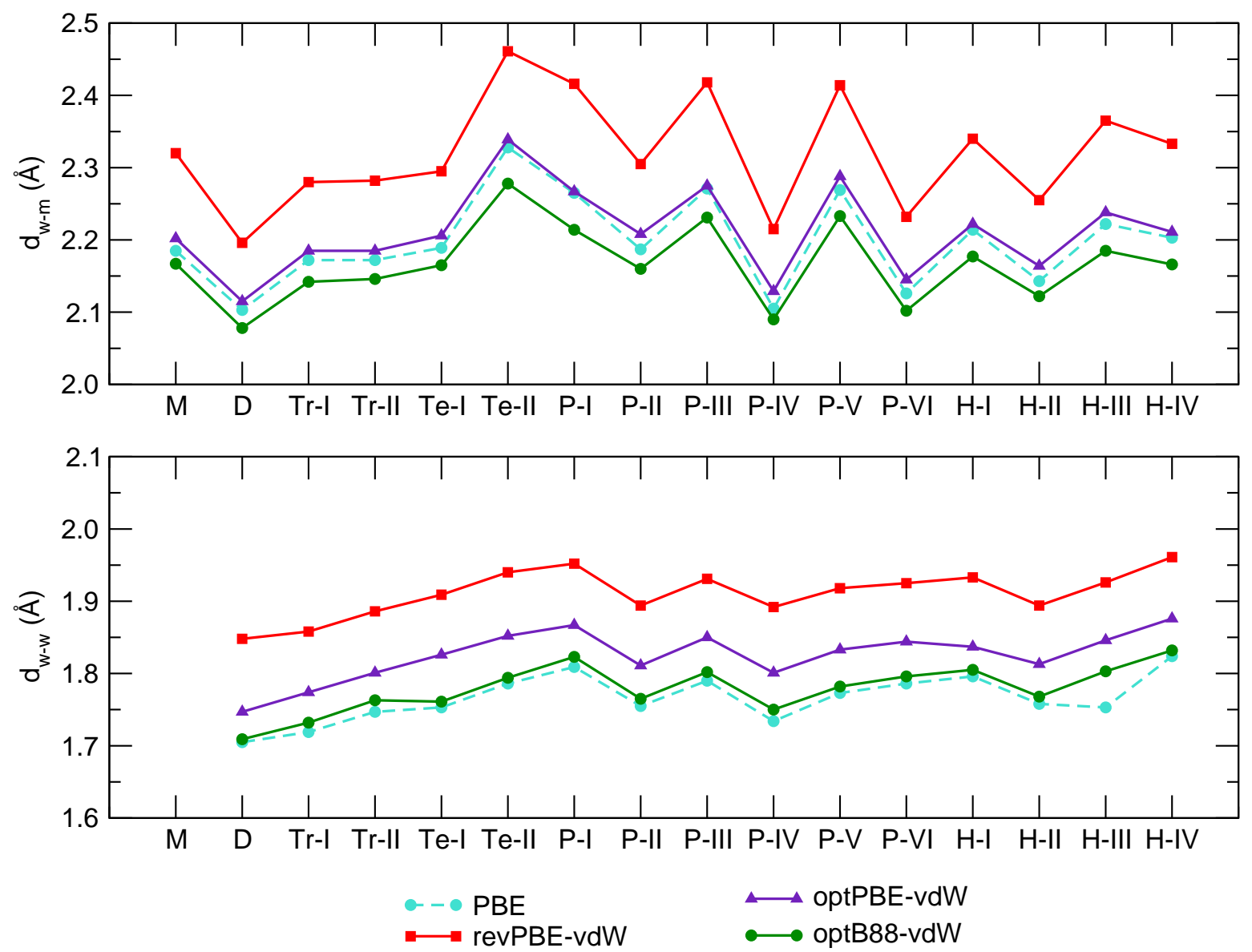

FIG. 5: Averaged nearest neighbor water-metal, $d_{\mathrm{w}-\mathrm{m}}$ (top), and water-water, $d_{\mathrm{w}-\mathrm{w}}$ (bottom), distances for the water clusters depicted in Fig. 3 using PBE, revPBE-vdW, optPBE-vdW, and optB88-vdW. The lines connecting the points are there to guide the eye. 
TABLE I: Adsorption energies (in $\mathrm{meV} / \mathrm{H}_{2} \mathrm{O}$ ) of an isolated water monomer on three different metal surfaces with PBE and the optB88-vdW functionals. Six different adsorption sites and adsorbed water geometries are shown (see Fig. 1). S1 is the most stable adsorption structure on all surfaces with all functionals. Negative adsorption energies correspond to favorable (exothermic) adsorption.

\begin{tabular}{lcccccc}
\hline \hline & $\mathrm{S} 1$ & $\mathrm{~S} 2$ & $\mathrm{~S} 3$ & $\mathrm{~S} 4$ & $\mathrm{~S} 5$ & $\mathrm{~S} 6$ \\
\hline $\mathrm{PBE}$ & & & & & & \\
\hline $\mathrm{Ag}(111)$ & -137 & -134 & -41 & -1 & 3 & 25 \\
$\mathrm{Au}(111)$ & -123 & -122 & -68 & 30 & 8 & 62 \\
$\mathrm{Ru}(0001)$ & -360 & -356 & -121 & -130 & -17 & -17 \\
\hline $\mathrm{optB} 88-\mathrm{vdW}$ & & & & & & \\
\hline $\mathrm{Ag}(111)$ & -281 & -280 & -195 & -151 & -117 & -103 \\
$\mathrm{Au}(111)$ & -281 & -281 & -213 & -120 & -122 & -76 \\
$\mathrm{Ru}(0001)$ & -541 & -538 & -271 & -334 & -131 & -125 \\
\hline \hline
\end{tabular}


TABLE II: Adsorption energies $\left(E_{\text {ads }}\right.$ in $\left.\mathrm{meV} / \mathrm{H}_{2} \mathrm{O}\right)$ and the optimized distance between the $\mathrm{O}$ atom of a water molecule and the nearest metal atom on the surface $\left(d_{\mathrm{w}-\mathrm{m}}\right.$ in $\left.\AA\right)$ for water monomers at the equilibrium adsorption site (S1 in Fig. 1) on all the metal surfaces investigated. Results for the PBE, revPBE-vdW, and optB88-vdW functionals are reported.

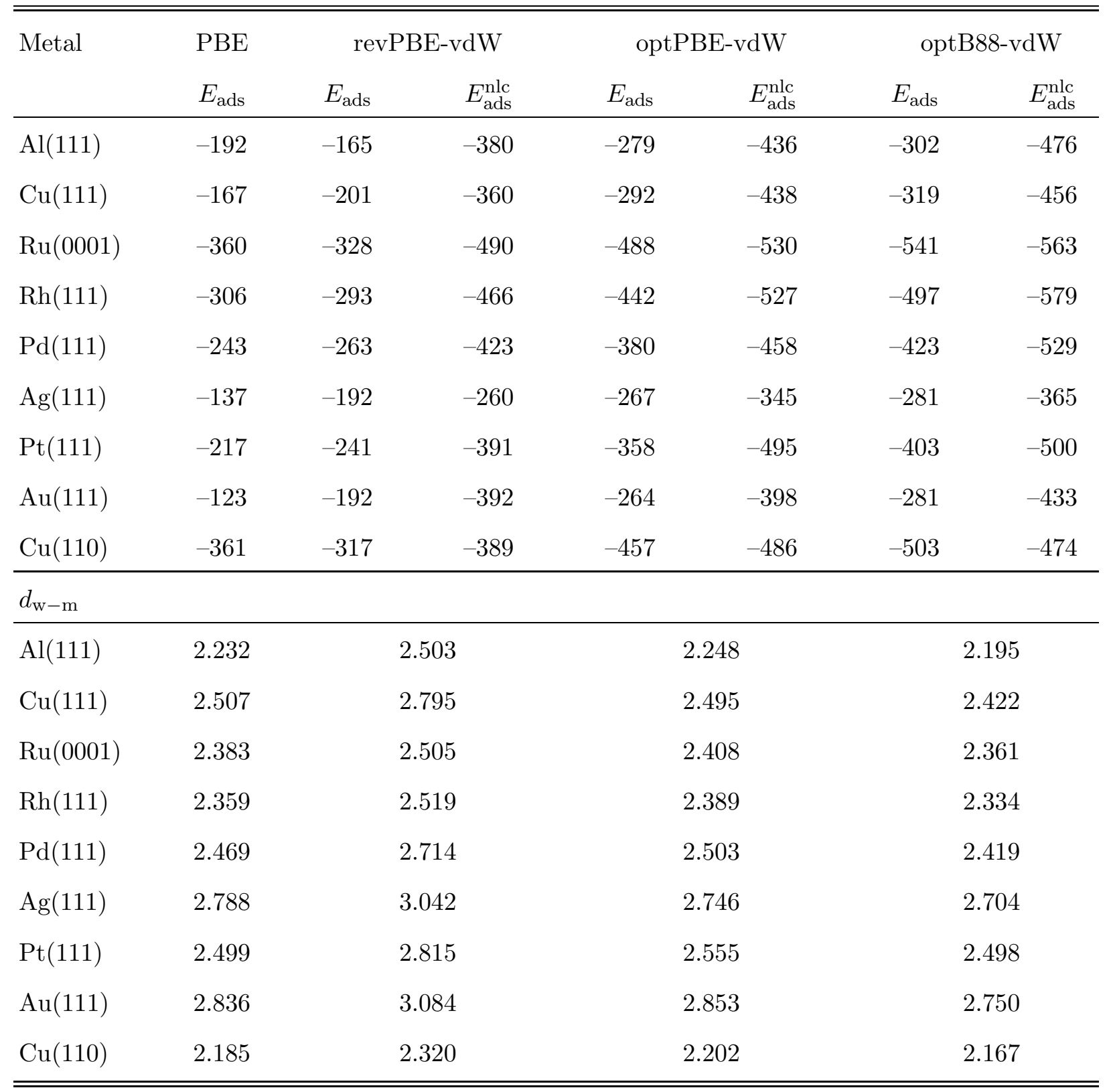


TABLE III: Computed adsorption energies $\left(E_{\text {ads }}\right)$ of water monomer $(\mathrm{M})$, dimer $(\mathrm{D})$, trimers $(\mathrm{Tr})$, tetramers $(\mathrm{Te})$, pentamers $(\mathrm{P})$, and hexamers $(\mathrm{H})$ adsorbed on $\mathrm{Cu}(110)$ using $\mathrm{PBE}$, revPBEvdW, optPBE-vdW, and optB88-vdW DFs. Water-water $\left(E_{\mathrm{gas}}^{\mathrm{ww}}\right)$ and water-metal $\left(E_{\mathrm{ads}}^{\mathrm{wm}}\right)$ bonding contributions are also given. All values are in $\mathrm{meV} / \mathrm{H}_{2} \mathrm{O}$.

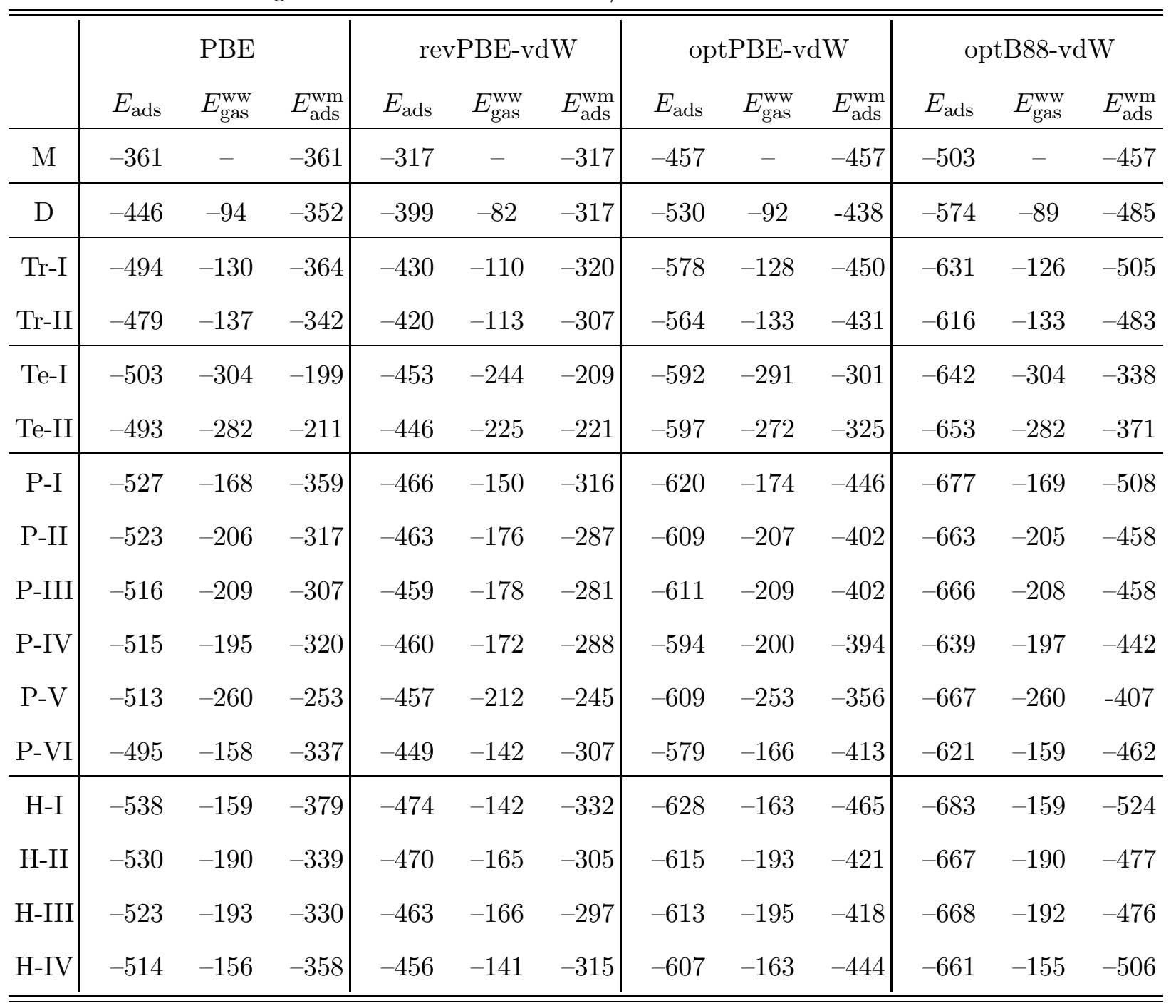


TABLE IV: Averaged nearest neighbor water-metal $\left(d_{\mathrm{w}-\mathrm{m}}\right)$ and H-bond $\left(d_{\mathrm{w}-\mathrm{w}}\right)$ distances for different water clusters adsorbed on $\mathrm{Cu}(110)$ using PBE, revPBE-vdW, optPBE-vdW, and optB88vdW DFs. All values are in $\AA$.

\begin{tabular}{|c|c|c|c|c|c|c|c|c|}
\hline & \multicolumn{2}{|c|}{ PBE } & \multicolumn{2}{|c|}{ revPBE-vdW } & \multicolumn{2}{|c|}{ optPBE-vdW } & \multicolumn{2}{|c|}{ optB88-vdW } \\
\hline & $d_{\mathrm{w}-\mathrm{m}}$ & $d_{\mathrm{w}-\mathrm{w}}$ & $d_{\mathrm{w}-\mathrm{m}}$ & $d_{\mathrm{w}-\mathrm{w}}$ & $d_{\mathrm{w}-\mathrm{m}}$ & $d_{\mathrm{w}-\mathrm{w}}$ & $d_{\mathrm{w}-\mathrm{m}}$ & $d_{\mathrm{w}-\mathrm{w}}$ \\
\hline M & 2.185 & - & 2.320 & - & 2.202 & - & 2.167 & - \\
\hline $\mathrm{D}$ & 2.103 & 1.705 & 2.196 & 1.848 & 2.115 & 1.747 & 2.078 & 1.709 \\
\hline $\operatorname{Tr}-\mathrm{I}$ & 2.172 & 1.719 & 2.280 & 1.858 & 2.185 & 1.774 & 2.142 & 1.732 \\
\hline Tr-II & 2.172 & 1.747 & 2.282 & 1.886 & 2.185 & 1.801 & 2.146 & 1.763 \\
\hline Te-I & 2.189 & 1.753 & 2.295 & 1.909 & 2.206 & 1.826 & 2.165 & 1.761 \\
\hline Te-II & 2.328 & 1.786 & 2.461 & 1.940 & 2.339 & 1.852 & 2.278 & 1.794 \\
\hline P-I & 2.265 & 1.809 & 2.416 & 1.952 & 2.267 & 1.867 & 2.214 & 1.823 \\
\hline P-II & 2.187 & 1.755 & 2.305 & 1.894 & 2.208 & 1.811 & 2.160 & 1.765 \\
\hline P-III & 2.271 & 1.790 & 2.418 & 1.931 & 2.275 & 1.850 & 2.231 & 1.802 \\
\hline P-IV & 2.105 & 1.734 & 2.215 & 1.892 & 2.129 & 1.801 & 2.090 & 1.750 \\
\hline $\mathrm{P}-\mathrm{V}$ & 2.269 & 1.773 & 2.414 & 1.918 & 2.288 & 1.833 & 2.233 & 1.782 \\
\hline P-VI & 2.126 & 1.786 & 2.232 & 1.925 & 2.145 & 1.844 & 2.102 & 1.796 \\
\hline H-I & 2.214 & 1.796 & 2.340 & 1.933 & 2.222 & 1.837 & 2.177 & 1.805 \\
\hline H-II & 2.143 & 1.758 & 2.255 & 1.894 & 2.164 & 1.813 & 2.122 & 1.768 \\
\hline H-III & 2.222 & 1.753 & 2.365 & 1.926 & 2.238 & 1.846 & 2.185 & 1.803 \\
\hline H-IV & 2.203 & 1.824 & 2.333 & 1.961 & 2.211 & 1.876 & 2.166 & 1.832 \\
\hline
\end{tabular}

